Commun. math. Phys. 28, 331-348 (1972)

(C) by Springer-Verlag 1972

\title{
Fields, Statistics and Non-Abelian Gauge Groups
}

\author{
Sergio Doplicher \\ Istituto Nazionale di Fisica Nucleare - Sezione di Roma \\ Istituto di Fisica "G. Marconi", Università di Roma \\ John E. Roberts \\ II. Institut für Theoretische Physik der Universität Hamburg
}

Received August 1, 1972

\begin{abstract}
We examine field theories with a compact group $\mathscr{G}$ of exact internal gauge symmetries so that the superselection sectors are labelled by the inequivalent irreducible representations of $\mathscr{G}$. A particle in one of these sectors obeys a parastatistics of order $d$ if and only if the corresponding representation of $\mathscr{G}$ is $d$-dimensional. The correspondence between representations of the observable algebra and representations of $\mathscr{G}$ extends to a mapping of the intertwining operators for these representations preserving linearity, tensor products and conjugation. Although we assume no explicit commutation property between fields, the commutation relations of fields of the same irreducible tensor character under $\mathscr{G}$ at spacelike separations are largely determined by the statistics parameter of the corresponding sector. For fields of conjugate irreducible tensor character the observable part of the commutator (anticommutator) vanishes at spacelike separations if the corresponding sector has para-Bose (para-Fermi) statistics.
\end{abstract}

\section{Introduction}

In studying the superselection structure of elementary particle physics one may distinguish the class of simple sectors. A simple sector is a superselection sector whose statistics is ordinary Bose or Fermi statistics. The set of simple sectors has the structure of a discrete Abelian group $\hat{\mathscr{G}}$ and one knows [1] that one can describe the sectors in a manner familiar from field theory by introducing unobservable quantities. Thus there is a field algebra $\mathfrak{F}$ made up of Bose and Fermi fields and a gauge group $\mathscr{G}$, the dual group of $\hat{\mathscr{G}}$, which allows us to recover the observable algebra as the gauge-invariant part of $\mathfrak{F}$.

We recall that $\mathfrak{F}$ is generated by the observable algebra together with a certain group of unitaries from $\mathfrak{F}$ called the field group. It was thus enough to construct a field group as a abstract group and this turned out to be equivalent to a standard problem in the theory of group extensions.

If we now consider, more generally, the set of finite sectors defined in [2] and therefore seek to include the sectors with parastatistics there is every reason to believe that a similar result should hold. Indeed, we

23 Commun. math. Phys., Vol. 28 
know [2] that the set of finite sectors has the same structure as the spectrum $^{1}$ of a compact group because we have a product of sectors, a conjugation of sectors and a statistical dimension for sectors.

Constructing a field algebra in this case is a much more difficult problem and the main aim of this paper is to pose the problem correctly. To do this we shall return to the setting of [3] and suppose that we have been given a field algebra on a Hilbert space $\mathscr{H}$ and a gauge group from which the observable algebra is derived by the principle of gauge invariance. As further input we require fields commuting with the observables for spacelike separations, positivity of the energy in the form of the Reeh-Schlieder property of analytic vectors and duality for the observables in the vacuum sector. We shall state precisely only those assumptions which are actually used. For the general setting we refer to assumptions 1 to 6 of [3] and assumption 7 in the strengthened form of [3; Eq. (1.23)] ${ }^{2}$.

We know that under these assumptions states given by vectors in $\mathscr{H}$ are asymptotically indistinguishable from the vacuum for observations in far away regions of space. In other words the field algebra gives us a set of sectors satisfying criterion 1.1 of [2]. Consequently we may apply to these sectors the intrinsic notion of statistics described in [2]: they obey para-Bose or para-Fermi statistics of a certain order. We show here that this order is related to the gauge group. In the correspondence [3] between these sectors and the elements of the spectrum $\hat{\mathscr{G}}$ of the gauge group $\mathscr{G}$, the order of the parastatistics is just the dimension of the corresponding group representation.

We may describe the sectors given by ₹ in terms of localized morphisms [2] and are therefore led to consider a certain subset $\Delta^{\prime}$ of localized morphisms ${ }^{3}$. As the field group in [1] consisted of the unitary operators inducing localized automorphisms of $\mathfrak{A}$ we introduce the set $H(\varrho)$ of strictly local elements $\psi$ of $\mathfrak{F}$ which induce a localized morphism $\varrho \in \Delta^{\prime}$, in the sense that

$$
\psi A=\varrho(A) \psi, \quad A \in \mathfrak{A} .
$$

Every element $\psi$ of $H(\varrho)$ is a multiple of an isometry and its adjoint $\psi^{*}$ acting on the vacuum creates a strictly localized state of $\mathfrak{A}$,

$$
\frac{\left(\psi^{*} \Omega, A \psi^{*} \Omega\right)}{\left(\Omega, \psi \psi^{*} \Omega\right)}=\omega_{0} \circ \varrho(A), \quad A \in \mathfrak{A}, \quad 0 \neq \psi \in H(\varrho) .
$$

${ }^{1}$ The term spectrum is used to denote the set of equivalence classes of irreducible representations. The structure referred to in the text is of course the tensor product and complex conjugation of representations and the dimension of a representation.

${ }^{2}$ It should be emphasized that these assumptions are fulfilled in models derived by taking a tensor product of copies of the free field algebra with a gauge group acting on the internal degrees of freedom thus introduced into the theory.

${ }^{3}$ There might be other superselection quantum numbers besides those carried by field operators from $\mathfrak{F}$. 
The set $H\left(\Delta^{\prime}\right)=\bigcup_{\varrho \in \Delta^{\prime}} H(\varrho)$ is the analogue of the field group and we show in particular that $H\left(\Delta^{\prime}\right)$ generates $\mathfrak{F}$.

We are now able to be more explicit about how the representations of $\mathfrak{A}$ are linked to representations of the gauge group. The gauge automorphisms induce a unitary representation $D(\varrho)$ of $\mathscr{G}$ on $H(\varrho)$. Furthermore an intertwiner $\boldsymbol{T}$ from $\varrho_{1}$ to $\varrho_{2}$ induces a linear mapping $D(\boldsymbol{T})$ from $H\left(\varrho_{1}\right)$ to $H\left(\varrho_{2}\right)$ which interwines the representations $D\left(\varrho_{1}\right)$ and $D\left(\varrho_{2}\right)$. The correspondence $D$ provides the link between the representations of $\mathfrak{A}$ and those of $\mathscr{G}$; it is linear and preserves adjoints, direct sums and tensor products. Furthermore the dimension of $D(\varrho)$ is just the statistical dimension of the localized morphism $\varrho$. In fact constructing such a correspondence $D$ is the key to the problem of constructing a field algebra and is the analogue of the group extension problem solved in [1].

We remind the reader that we do not assume any explicit commutation relations between charged fields at spacelike separations. In Sections IV and $\mathrm{V}$ we study to what extent these properties are determined by the statistics of the corresponding superselection sectors. In the first place, there is a direct connexion between the "observable part" of a commutator or anticommutator and the para-Bose or para-Fermi character of the sector involved. To get a non-zero "observable part" we must combine a field operator $F_{1}$ of irreducible tensor character $\sigma$ with a field operator $F_{2}$ of tensor character $\bar{\sigma}$. We find (Theorem 4.2) that

$$
\int \alpha_{g}\left(F_{1} F_{2} \mp F_{2} F_{1}\right) d \mu(g)=0
$$

if $F_{1}$ and $F_{2}$ are spacelike separated, where the - or + sign is taken according to whether the sector corresponding to $\sigma$ has para-Bose or para-Fermi statistics.

Another connexion between statistics and commutation properties is found by studying the effect of "wave function permutations" on products of $\psi_{j} \in H\left(\varrho_{j}\right)$ where the $\varrho_{j}$ are mutually spacelike and lead to the same sector. If the $\psi_{j}$ commute or anticommute with each other according to whether the sector obeys para-Bose or para-Fermi statistics, wave function permutations and permutations of the gauge indices differ only by a sign. This is the case of normal commutation relations. In the general case we show that these two representations of the permutation group may differ in addition by a unitary equivalence. We leave open the problem of whether this unitary equivalence can be removed by a Klein transformation. 


\section{Creation Operators for Strictly Localized States}

We recall briefly our notation. The algebra $\mathfrak{A}$ of quasilocal observables consists of the gauge-invariant elements of the irreducible $C^{*}$-algebra $\mathfrak{F}$ of quasilocal fields:

$$
\mathfrak{U}=\mathfrak{F} \cap \mathscr{U}(\mathscr{G})^{\prime} ;^{4}
$$

$\mathbb{F}$ is the uniform closure of the union of von Neumann algebras $\mathbb{F}(\mathcal{O})$ of field operators belonging to the double cone $\mathcal{O} \in \mathscr{K}^{5}$ in space-time, and the local algebras of observables

$$
\mathfrak{A}(\mathcal{O})=\mathfrak{F}(\mathcal{O}) \cap \mathscr{U}(\mathscr{G})^{\prime}
$$

generate $\mathfrak{A}$ in the same way. In fact $\mathfrak{A}$ is the image of $\mathfrak{F}$ under the normal projection map $m$ of $\mathscr{B}(\mathscr{H})$ onto $\mathscr{U}(\mathscr{G})^{\prime}$ given by

$$
m(B)=\int_{\mathscr{G}} \mathscr{U}(g) B \mathscr{U}(g)^{-1} d \mu(g)
$$

where $\mu$ is the normalized Haar measure on the compact gauge group $\mathscr{G}$; consequently, $\mathfrak{I}^{\prime}=\mathscr{U}(\mathscr{G})^{\prime \prime}$ (see [3; Section III]).

The following two assumptions are central to this section: first a "duality" relation [1; Eq. (1.23)]

a) $m\left(\mathfrak{F}(\mathcal{O})^{\prime}\right)=\mathfrak{A}\left(\mathcal{O}^{\prime}\right)^{-}, \mathcal{O} \in \mathscr{K}$,

where $\mathfrak{A}\left(\mathcal{O}^{\prime}\right)$ is the $C^{*}$-algebra generated by the $\mathfrak{A}\left(\mathcal{O}_{\lambda}\right)$, where $\mathcal{O}_{\lambda} \in \mathscr{K}$ is spacelike to $\mathcal{O}$. The second property follows from the Reeh-Schlieder theorem: vectors analytic for the energy are cyclic and separating for each $\mathfrak{F}(\mathcal{O})$. Specializing to the vacuum state vector $\Omega$ we assume

b) $\mathfrak{F}(\mathcal{O}) \Omega$ and $\mathscr{F}(\mathcal{O})^{\prime} \Omega$ are dense in $\mathscr{H}$.

Note that a) is a strong form of the requirement that fields should commute locally with observables. It also implies the basic duality property of observables in the vacuum sector:

$$
\pi_{0}\left(\mathfrak{A}\left(\mathcal{O}^{\prime}\right)\right)^{\prime}=\pi_{0}(\mathfrak{A}(\mathcal{O})), \quad \mathcal{O} \in \mathscr{K}
$$

where $\pi_{0}$ is the irreducible subrepresentation of $\mathfrak{A}$ acting on the subspace $\mathscr{H}_{0}$ of $\mathscr{G}$-invariant vectors [ 3 ; Section IV]. $\pi_{0}$ is generated by the vacuum state $\omega_{0}: \omega_{0}(A)=(\Omega, A \Omega), A \in \mathfrak{A}$.

As mentioned in the Introduction, if $\pi$ is an irreducible representation of $\mathfrak{A}$ appearing in the decomposition of the defining representation of $\mathfrak{A}$ on $\mathscr{H}$ there is a localized morphism ${ }^{6} \varrho$ such that $\pi$ and $\pi_{0}{ }^{\circ} \varrho$ are unitarily

\footnotetext{
${ }^{4} \mathscr{S}^{\prime}$ denotes the commutant of a set $\mathscr{S}$ of bounded operators and $\mathscr{S}^{-}$its weak closure.

${ }^{5} \mathscr{O} \in \mathscr{K}$ if $\mathcal{O}$ is the intersection of a closed forward and a closed backward light cone having an interior point in common. $\mathcal{O}^{\prime}$ denotes the open set of points spacelike to $\mathcal{O}$.

${ }^{6} \mathrm{~A}$ morphism $\varrho$ of the $C^{*}$-algebra $\mathfrak{A}$ into itself is said to be localized in $\mathscr{O} \in \mathscr{K}$ if $\varrho(A)=A$ for $A \in \mathfrak{U}\left(\mathcal{O}^{\prime}\right)$.
} 
equivalent ${ }^{7}$. If $\varrho$ is such a morphism, there are vectors $\Phi \in \mathscr{H}$ such that

$$
(\Phi, A \Phi)=\omega_{0} \circ \varrho(A), \quad A \in \mathfrak{A} .
$$

These are precisely the unit vectors in a subspace $E_{\varrho} \mathscr{H}$, where $E_{\varrho}$ is a minimal projection in $\mathfrak{U}^{\prime \prime}=\mathscr{U}(\mathscr{G})^{\prime}$.

2.1. Proposition. Let $\varrho$ be an irreducible morphism localized in $\mathcal{O}$. For each $\Phi \in E_{\varrho} \mathscr{H}$ there is a field operator $\psi \in \mathfrak{F}(\mathcal{O})$ such that

$$
\begin{gathered}
\psi^{*} \Omega=\Phi \\
\psi A=\varrho(A) \psi, \quad A \in \mathfrak{A} .
\end{gathered}
$$

Proof. From Eq. (2.3) we have

$$
(\Phi, A \Phi)=(\Omega, A \Omega), \quad A \in \mathfrak{H}\left(\mathcal{O}^{\prime}\right)^{-} .
$$

For each $F \in \mathfrak{F}(\mathcal{O})^{\prime}$ we have by assumption a) and (2.3)

Hence

$$
E_{\varrho} m\left(F^{*} F\right) E_{\varrho}=\omega_{0}^{\circ} m\left(F^{*} F\right) E_{\varrho}=\|F \Omega\|^{2} E_{\varrho} .
$$

$$
\|F \Phi\|^{2} \leqq \operatorname{Tr} E_{\varrho} F^{*} F E_{\varrho}=\operatorname{Tr} E_{\varrho} m\left(F^{*} F\right) E_{\varrho}=d\|F \Omega\|^{2}
$$

where $d$ is the (finite) dimension of $E_{\varrho}$. As $\mathfrak{F}(\mathcal{O})^{\prime} \Omega$ is a dense linear subset of $\mathscr{H}$, there is a unique bounded operator $\psi^{*}$ on $\mathscr{H}$ such that

$$
\psi^{*} F \Omega=F \Phi, \quad F \in \mathfrak{F}(\mathcal{O})^{\prime} ;
$$

Clearly $\|\psi\| \leqq \sqrt{d}$ and $\psi \in \mathfrak{F}(\mathcal{O})^{\prime \prime}=\mathfrak{F}(\mathcal{O})$. By (2.3) we can also define on $\mathscr{H}_{0}$ an isometric map taking $\varrho(A) \Omega$ to $A \Phi, A \in \mathfrak{A}$. Since this map agrees with $\psi^{*}$ on the set $\mathfrak{A}\left(\mathcal{O}^{\prime}\right) \Omega$ dense in $\mathscr{H}_{0}$, it agrees with $\psi^{*}$ everywhere on $\mathscr{H}_{0}$, i.e.

$$
\psi^{*} \varrho(A) \Omega=A \psi^{*} \Omega, \quad A \in \mathfrak{A} .
$$

If $A$ is a local observable we deduce from assumption b) that $\psi^{*} \varrho(A)=A \psi^{*}$. Hence by norm continuity this holds for any $A \in \mathfrak{A}$ and taking adjoints we get (2.5).

The properties of field operators fulfilling (2.5) will be studied in the next section. Here we only note that the operator $m\left(\psi \psi^{*}\right)$ belongs to $\mathfrak{A}(\mathcal{O})$ and commutes with $\varrho(\mathfrak{U})$ by $(2.5)$; consequently it is a multiple of the identity. Since $\left(\Omega, m\left(\psi \psi^{*}\right) \Omega\right)=\left\|\psi^{*} \Omega\right\|^{2}=\|\Phi\|^{2}$ we have for a unit vector $\Phi$,

$$
m\left(\psi \psi^{*}\right)=I .
$$

7 This follows from [3; Theorem 6.1] and [2; Proposition 1.2]. The former result requires that every subspace stable under $\mathfrak{A}$ contains a cyclic and separating vector for $\mathfrak{F}(\mathcal{O})$. This is of course a consequence of the Reeh-Schlieder Theorem. 
2.2. Proposition. If $F \in \mathfrak{F}(\mathcal{O})$ is an irreducible tensor under the gauge group, then

$$
F=B \psi
$$

where $B \in \mathfrak{A}(\mathcal{O})$ and $\psi \in \mathfrak{F}(\mathcal{O})$ fulfills (2.5) for some $\varrho$.

Proof. The vector $F^{*} \Omega$ belongs to an irreducible subspace under $\mathfrak{A}$. Since the corresponding subrepresentation of $\mathfrak{A}$ is equivalent to $\pi_{0} \circ \varrho$ where $\varrho$ is a morphism localized in $\mathcal{O}$, there is a vector $\Phi \in \mathscr{H}_{0}$ such that

$$
\left(F^{*} \Omega, A F^{*} \Omega\right)=(\Phi, \varrho(A) \Phi), \quad A \in \mathfrak{A},
$$

Since $\|A \Phi\|=\left\|F^{*} A \Omega\right\|$ for $A \in \mathfrak{A}\left(\mathcal{O}^{\prime}\right)$, we can define a bounded operator $T$ on $\mathscr{H}_{0}$ by

$$
T A \Omega=A \Phi, \quad A \in \mathfrak{A}\left(\mathcal{O}^{\prime}\right) .
$$

Since $T$ commutes with $\mathfrak{A}\left(\mathcal{O}^{\prime}\right)$ on $\mathscr{H}_{0}$ we see from (2.2) that $T$ is the restriction to $\mathscr{H}_{0}$ of an operator $B^{*} \in \mathfrak{A}(\mathcal{O})$, so

$$
\Phi=B^{*} \Omega .
$$

Further by Proposition 2.1 there is a field operator $\psi$ satisfying (2.5) and (2.6) and leading from $\mathscr{H}_{0}$ to $\overline{\mathfrak{A} F^{*} \Omega}$. The vectors $F^{*} \Omega$ and $\psi^{*} B^{*} \Omega$ belong to the same irreducible subspace under $\mathfrak{A}$ and induce the same state on $\mathfrak{A}$ since, using (2.5), (2.6) and (2.8),

$$
\begin{aligned}
\left(\psi^{*} B^{*} \Omega, A \psi^{*} B^{*} \Omega\right) & =\left(B^{*} \Omega, \varrho(A) \psi \psi^{*} B^{*} \Omega\right)=\left(\Phi, \varrho(A) m\left(\psi \psi^{*}\right) \Phi\right) \\
& =(\Phi, \varrho(A) \Phi)=\left(F^{*} \Omega, A F^{*} \Omega\right) .
\end{aligned}
$$

Modifying $B$ by a phase if needed, we conclude that

$$
F^{*} \Omega=\psi^{*} B^{*} \Omega
$$

and by assumption b) we deduce (2.7).

Remark 1. Irreducible tensors $F_{\sigma} \in \mathfrak{F}(\mathcal{O})$ are obtained from any $F \in \mathfrak{F}(\mathcal{O})$ by averaging $\alpha_{g}(F)=\mathscr{U}(g) F \mathscr{U}(g)^{-1}$ with a coefficient $\left(\phi_{1}, u_{\sigma}(g) \phi_{2}\right)$ of the irreducible representation $\sigma$ :

$$
F_{\sigma}=\int \overline{\left(\phi_{1}, u_{\sigma}(g) \phi_{2}\right)} \alpha_{g}(F) d \mu(g)
$$

where the integral converges in the weak topology. It follows that the linear span of irreducible tensors in $\mathfrak{F}(\mathcal{O})$ is weakly dense in $\mathfrak{F}(\mathcal{O})$. To see this recall that the finite linear combinations of coefficients of irreducible representations of a compact group $\mathscr{G}$ form a subspace $\mathscr{V}$ uniformly dense in the space $\mathscr{C}(\mathscr{G})$ of all continuous functions on $\mathscr{G}$. Hence we may approximate the Dirac measure at the identity weakly by functions from $\mathscr{V}$. In other words there is a net of functions $h_{\alpha} \in \mathscr{V}$ such that

$$
f(e)=\lim _{\alpha} \int_{\mathscr{G}} h_{\alpha}(g) f(g) d \mu(g), \quad f \in \mathscr{C}(\mathscr{G}) .
$$


Taking $f$ to be the continuous function $\left(\Psi_{1}, \alpha_{g}(\mathrm{~F}) \Psi_{2}\right)$ with $\Psi_{1}, \Psi_{2} \in \mathscr{H}$, $F \in \mathfrak{F}(\mathcal{O})$, we get

where

$$
F=\text { weak } \lim _{\alpha} F_{\alpha}
$$

$$
F_{\alpha}=\int h_{\alpha}(g) \alpha_{g}(F) d \mu(g) \in \mathfrak{F}(\mathcal{O})
$$

is a finite linear combination of irreducible tensors under $\mathscr{G}$.

Remark 2. Since $\mathfrak{A}(\mathcal{O})$ is a $C^{*}$-algebra with identity, the operator $B$ of Proposition 2.2 is a linear combination of four unitaries $U_{j} \in \mathfrak{A}(\mathcal{O})$, $j=1, \ldots, 4$; setting $\psi_{j}=U_{j} \psi$ we see that

$$
\psi_{j} A=\varrho_{j}(A) \psi_{j}, \quad A \in \mathfrak{A}
$$

where $\varrho_{j}=\sigma_{U_{j}} \varrho$, i.e. $\varrho_{j}(A)=U_{j} \varrho(A) U_{j}^{-1}$, is again a morphism localized in $\mathcal{O}$. Hence $F$ is a linear combination of four field operators each satisfying (2.5).

Combining the last statements we get

2.3. Theorem. The linear span of the field operators satisfying (2.5) where $\varrho$ varies in $\Delta(\mathcal{O})$, the set of morphisms localized in $\mathcal{O}$, is weakly dense in the von Neumann algebra $\mathfrak{F}(\mathcal{O})$.

\section{Intertwiners and the Gauge Group}

For a localized morphism $\varrho$ we write

$$
H(\varrho)=\left\{\psi \in \bigcup_{\mathcal{O}} \mathfrak{F}(\mathcal{O}): \psi A=\varrho(A) \psi, \quad A \in \mathfrak{A}\right\}
$$

From Proposition 2.1 we know that if $\pi_{0} \circ \varrho$ is an irreducible subrepresentation of the defining representation of $\mathscr{A}$ on $\mathscr{H}$ then $H(\varrho) \neq\{0\}$. On the other hand the converse is also true since $0 \neq \psi \in H(\varrho)$ implies $0 \neq \psi^{*} \Omega \in E_{\varrho} \mathscr{H}$ by the remarks following Proposition 2.1. The properties of the subspace $H(\varrho)$ motivate the following definition:

3.1. Definition. A subspace $H \subset \mathscr{B}(\mathscr{H})$ is called a subspace of isometries if every element of $H$ is a multiple of an isometry.

If $H$ is a subspace of isometries then the norm on $H$ is induced by a scalar product $($,$) and we have$

$$
\psi_{1}^{*} \psi_{2}=\left(\psi_{1}, \psi_{2}\right) I, \quad \psi_{1}, \psi_{2} \in H .
$$

If $H_{1}$ and $H_{2}$ are subspace of $\mathscr{B}(\mathscr{H})$ we denote by $H_{1} H_{2}$ their inner tensor product, i.e. the linear subspace of $\mathscr{B}(\mathscr{H})$ generated by $\psi_{1} \psi_{2}$, $\psi_{1} \in H_{1}, \psi_{2} \in \mathrm{H}_{2}$. If $H_{1}$ and $H_{2}$ are subspaces of isometries then $H_{1} H_{2}$ is a subspace of isometries. Further $H_{1} H_{2}^{*}$ may be naturally identified 
with the set of finite rank operators from $H_{2}$ to $H_{1}$, since given $\psi_{1} \in H_{1}$ and $\psi_{2}, \psi \in \mathrm{H}_{2}$ we have

$$
\psi_{1} \psi_{2}^{*} \psi=\left(\psi_{2}, \psi\right) \psi_{1} .
$$

We call the support of a subspace of isometries $H$, the smallest projection in $\mathscr{B}(\mathscr{H})$ such that $E \psi=\psi$, for all $\psi \in H$. It is clear that $E=\sum_{i} \psi_{i} \psi_{i}^{*}$ where $\psi_{i}$ runs over a maximal orthonormal set of $H$. Further $T \psi=0$ if and only if $T E=0$. Note that $E$ is also the smallest projection majorizing the projections of $H H^{*}$. If $I \in H H^{*}$ then $H$ is finite dimensional with support $I$ and is also a maximal subspace of isometries.

3.2. Proposition. Let $\varrho$ be a localized morphism then $H(\varrho)$ is a subspace of isometries of $\mathscr{B}(\mathscr{H})$.

Proof. If $\psi_{1}, \psi_{2} \in H(\varrho)$ then $\psi_{1}^{*} \psi_{2} \in \mathfrak{U}^{\prime}=\mathscr{U}(\mathscr{G})^{\prime \prime}$ by [3; Eq. (3.3)]. Hence $\psi_{1}^{*} \psi_{2} \Omega=\lambda \Omega$ and by assumption b), $\psi_{1}^{*} \psi_{2}=\lambda I$ showing that $H(\varrho)$ is a subspace of isometries.

We are not interested in all localized morphisms on $\mathfrak{A}$ here but only in an appropriate subset which could be used instead of the field algebra to describe the structure of sectors contained in the defining representation of $\mathfrak{A}$ on $\mathscr{H}$. It will turn out that this is just the subset $\Delta^{\prime}$ defined by

$$
\Delta^{\prime}=\left\{\varrho \in \Delta: I \in H(\varrho) H(\varrho)^{*}\right\} .
$$

3.3. Proposition. If $\varrho_{1}, \varrho_{2} \in \Delta^{\prime}$ then $\varrho_{1} \varrho_{2} \in \Delta^{\prime}$ and $H\left(\varrho_{1} \varrho_{2}\right)=H\left(\varrho_{1}\right) H\left(\varrho_{2}\right)$. Further $\varrho \in \Delta^{\prime}$ if and only if $\varrho$ is a finite direct sum of irreducibles from $\Delta^{\prime}$.

Proof. We have trivially $H\left(\varrho_{1}\right) H\left(\varrho_{2}\right) \cong H\left(\varrho_{1} \varrho_{2}\right)$. If $\varrho_{1}, \varrho_{2} \in \Delta^{\prime}$ then $I \in H\left(\varrho_{i}\right) H\left(\varrho_{i}\right)^{*}, i=1,2$. Hence $I \in H\left(\varrho_{1}\right) H\left(\varrho_{2}\right) H\left(\varrho_{2}\right)^{*} H\left(\varrho_{1}\right)^{*}$ showing that $H\left(\varrho_{1}\right) H\left(\varrho_{2}\right)$ is a maximal subspace of isometries. Thus $H\left(\varrho_{1}\right) H\left(\varrho_{2}\right)$ $=H\left(\varrho_{1} \varrho_{2}\right)$ and $\varrho_{1} \varrho_{2} \in \Delta^{\prime}$. Now if $\boldsymbol{T}=\left(\varrho_{1}|T| \varrho_{2}\right)^{8}$ and $\psi \in H\left(\varrho_{2}\right)$ then

$$
T \psi A=T \varrho_{2}(A) \psi=\varrho_{1}(A) T \psi, \quad A \in \mathfrak{A}
$$

thus $T \psi \in H\left(\varrho_{1}\right)$. Letting $\mathscr{T}\left(\varrho_{2}, \varrho_{1}\right)$ denote the set of intertwiners from $\varrho_{2}$ to $\varrho_{1}$ we have

$$
\begin{gathered}
T H\left(\varrho_{2}\right) \subseteq H\left(\varrho_{1}\right), \quad \boldsymbol{T} \in \mathscr{T}\left(\varrho_{2}, \varrho_{1}\right), \\
\mathscr{T}\left(\varrho_{2}, \varrho_{1}\right) H\left(\varrho_{2}\right) H\left(\varrho_{2}\right)^{*} \mathscr{T}\left(\varrho_{2}, \varrho_{1}\right)^{*} \cong H\left(\varrho_{1}\right) H\left(\varrho_{1}\right)^{*} .
\end{gathered}
$$

Eq. (3.7) shows that $\Delta^{\prime}$ is closed under finite direct sums and also under subrepresentations. From (3.6) we deduce

$$
T H(\varrho) \subseteq H(\varrho), \quad \boldsymbol{T} \in \mathscr{T}(\varrho, \varrho) .
$$

${ }^{8}$ We recall from $[2 ; \S \mathrm{IV}]$ that this notation means that $\boldsymbol{T}$ is an intertwiner from $\varrho_{2}$ to $\varrho_{1}$ i.e. that $T \varrho_{2}(A)=\varrho_{1}(A) T$ for $A \in \mathfrak{A}$. 
But if $\varrho \in \Delta^{\prime}, H(\varrho)$ has support $I$ and is finite dimensional so $\mathscr{T}(\varrho, \varrho)$ is also finite dimensional and $\varrho$ is a finite direct sum of irreducibles. This completes the proof.

We now specify the irreducibles of $\Delta^{\prime}$ by using the gauge group.

3.4. Proposition. If $H(\varrho) \neq\{0\}$ then $g \rightarrow \alpha_{g}(\psi)$ defines a continuous unitary representation $D(\varrho)$ of $\mathscr{G}$ on $H(\varrho)$. Further if $\varrho$ is irreducible then $\varrho \in \Delta^{\prime}$ and $D(\varrho)$ is irreducible.

Proof. If $\psi \in \mathscr{H}(\varrho)$ and $A \in \mathfrak{A}$ then

$$
\alpha_{g}(\psi) A=\alpha_{g}(\psi A)=\alpha_{g}(\varrho(A) \psi)=\varrho(A) \alpha_{g}(\psi)
$$

hence $\alpha_{g}(\psi) \in H(\varrho)$. Furthermore if $\psi_{1}, \psi_{2} \in H(\varrho)$ then

$$
\alpha_{g}\left(\psi_{1}^{*}\right) \alpha_{g}\left(\psi_{2}\right)=\alpha_{g}\left(\psi_{1}^{*} \psi_{2}\right)=\psi_{1}^{*} \psi_{2} .
$$

Thus $\psi \rightarrow \alpha_{g}(\psi)$ defines a unitary representation $D(\varrho)$ of $\mathscr{G}$ on $H(\varrho)$ which is clearly continuous since

$$
\left\|\psi-\alpha_{g}(\psi)\right\|=\|\psi \Omega-\mathscr{U}(g) \psi \Omega\| .
$$

Now the action of $\mathscr{G}$ respects the identification of $H(\varrho) H(\varrho)^{*}$ with the finite rank operators on $H(\varrho)$, since $\alpha_{g}$ is an automorphism. Thus the projection onto a subspace of $H(\varrho)$ on which $D(\varrho)$ acts irreducibly must correspond to a projection in $H(\varrho) H(\varrho)^{*} \cap \mathfrak{A}$. However $H(\varrho) H(\varrho)^{*} \varrho \varrho(\mathfrak{A})^{\prime}$ and if $\varrho$ is irreducible the only projections in $\mathfrak{A} \cap \varrho(\mathfrak{A})^{\prime}$ are 0 and $I$. Hence, if $\varrho$ is irreducible, $I \in H(\varrho) H(\varrho)^{*}, \varrho \in \Delta^{\prime}$ and $D(\varrho)$ is irreducible.

3.5. Proposition. Let $\varrho_{1}, \varrho_{2} \in \Delta^{\prime}$ then under the canonical isomorphism of $H\left(\varrho_{2}\right) H\left(\varrho_{1}\right)^{*}$ with the linear mappings from $H\left(\varrho_{1}\right)$ to $H\left(\varrho_{2}\right), \mathscr{T}\left(\varrho_{1}, \varrho_{2}\right)$ is mapped onto the set of intertwining operators from $D\left(\varrho_{1}\right)$ to $D\left(\varrho_{2}\right)$.

Proof. Eq. (3.6) shows that $\mathscr{T}\left(\varrho_{1}, \varrho_{2}\right) \cong H\left(\varrho_{2}\right) H\left(\varrho_{1}\right)^{*}$. Also

$$
T \alpha_{g}(\psi)=\alpha_{g}(T \psi), \quad \boldsymbol{T} \in \mathscr{T}\left(\varrho_{1}, \varrho_{2}\right), \quad \psi \in H\left(\varrho_{1}\right), \quad g \in \mathscr{G}
$$

so that $D(\boldsymbol{T})$, the image of $\boldsymbol{T}$ under the canonical isomorphism, interwines $D\left(\varrho_{1}\right)$ and $D\left(\varrho_{2}\right)$. Conversely suppose $S \in H\left(\varrho_{2}\right) H\left(\varrho_{1}\right)^{*}$ and that

then

$$
\begin{gathered}
S \alpha_{g}(\psi)=\alpha_{g}(S \psi), \\
\psi \in H\left(\varrho_{1}\right), \quad g \in \mathscr{G}
\end{gathered}
$$

$$
\begin{gathered}
S \psi=\alpha_{g}(S) \psi, \\
\psi \in H\left(\varrho_{1}\right), \quad g \in \mathscr{G}, \\
S \varrho_{1}(A) \psi=S \psi A=\varrho_{2}(A) S \psi, \\
A \in \mathfrak{A}, \quad \psi \in H\left(\varrho_{1}\right) .
\end{gathered}
$$


Since $H\left(\varrho_{1}\right)$ has support $I$, we deduce that

$$
\begin{gathered}
S=\alpha_{g}(S), \quad g \in \mathscr{G} \\
S \varrho_{1}(A)=\varrho_{2}(A) S, \quad A \in \mathfrak{A}
\end{gathered}
$$

thus $\boldsymbol{S} \in \mathscr{T}\left(\varrho_{1}, \varrho_{2}\right)$ completing the proof.

From the last two results we see how the representations of $\mathfrak{A}$ are linked to those of $\mathscr{G}$. A localized morphism $\varrho \in \Delta^{\prime}$ provides on the one side a representation $\pi_{0} \circ \varrho$ and on the other a continuous unitary representation $D(\varrho)$ of $\mathscr{G}$. In the same way an intertwiner $\boldsymbol{T}=\left(\varrho_{2}|T| \varrho_{1}\right)$ provides an intertwiner $\pi_{0}(T)$ between the representations $\pi_{0}^{\circ} \varrho_{1}$ and $\pi_{0}{ }^{\circ} \varrho_{2}$ and also an intertwiner $D(T)$ between $D\left(\varrho_{1}\right)$ and $D\left(\varrho_{2}\right)$. The most potent way of expressing the properties of this correspondence $D$ is to draw on notions from category theory.

Let $\mathfrak{U}(\mathscr{G})$ denote the category of finite dimensional continuous unitary representations of the compact group $\mathscr{G}$. Thus the objects of $\mathfrak{u}(\mathscr{G})$ are the finite dimensional continuous unitary representations of $\mathscr{G}$ and the morphisms of $\mathfrak{U}(\mathscr{G})$ are the intertwining operators between these representations. The set of intertwining operators between two representations has the structure of a complex linear space and the adjoint operation is a conjugate linear contravariant involution leaving the objects invariant. $\mathfrak{U}(\mathscr{G})$ is furthermore a symmetric monoidal category ${ }^{9}$ with respect to the tensor product of representations. We let $\mathscr{T}^{\prime}$ denote the category whose objects are localized morphisms from $\Delta^{\prime}$ and whose morphisms are the intertwiners between these localized morphisms. We know from [2] that $\mathscr{T}^{\prime}$ has the same formal structure as $\mathfrak{U}(\mathscr{G})$ with the cross product playing the role of a tensor product.

3.6. Theorem. $D: \mathscr{T}^{\prime} \rightarrow \mathfrak{U}(\mathscr{G})$ is an equivalence of categories preserving the linear structure, adjoints and tensor products.

Proof. $D$ is clearly linear; further if $\boldsymbol{T}=\boldsymbol{T}_{1} \circ \boldsymbol{T}_{2}$ then $D(\boldsymbol{T})=D\left(\boldsymbol{T}_{1}\right) D\left(\boldsymbol{T}_{2}\right)$ hence $D$ is a functor. Also $D(\boldsymbol{T})^{*}=D\left(\boldsymbol{T}^{*}\right)$ so $D$ preserves adjoints. Given $\psi_{i} \in H\left(\varrho_{i}\right)$ and $\boldsymbol{T}_{i}=\left(\varrho_{i}^{\prime}\left|T_{i}\right| \varrho_{i}\right)$ for $i=1,2$ we have

$$
T_{1} \psi_{1} T_{2} \psi_{2}=T_{1} \varrho_{1}\left(T_{2}\right) \psi_{1} \psi_{2}
$$

Recalling the definition of the cross product of intertwiners [2; Eq. (4.5)] we have $\boldsymbol{T}_{1} \times \boldsymbol{T}_{2}=\left(\varrho_{1}^{\prime} \varrho_{2}^{\prime}\left|T_{1} \varrho_{1}\left(T_{2}\right)\right| \varrho_{1} \varrho_{2}\right)$ so that $D\left(\boldsymbol{T}_{1}\right) \otimes D\left(\boldsymbol{T}_{2}\right)=D\left(\boldsymbol{T}_{1} \times \boldsymbol{T}_{2}\right)$ and $D$ preserves tensor products. Now from Proposition 3.5 the functor $D$ is full and faithful, hence to show that $D$ is an equivalence it suffices to show [4; Theorem IV 4.1] that every representation of $\mathfrak{U}(\mathscr{G})$ is

9 The reader unfamiliar with category theory can consult for example Mac Lane [4] for the basic definitions used here. 
unitarily equivalent to some representation from $D\left(\mathscr{T}^{\prime}\right)$. Combining the remarks at the beginning of this section with Proposition 3.4 and [3; Theorem 3.6] we see that $D\left(\mathscr{T}^{\prime}\right)$ contains sufficient irreducibles. However $\mathscr{T}^{\prime}$ has direct sums by Proposition 3.3 and as $D$ is a functor preserving adjoints, $D\left(\mathscr{T}^{\prime}\right)$ also has direct sums. As every object of $\mathfrak{U}(\mathscr{G})$ is a finite direct sum of irreducibles this completes the proof.

\section{Statistics, Gauge Covariance and Commutation Properties}

From the analysis in [2] we know that a simple sector, i.e. one obeying Bose or Fermi statistics corresponds to a sector satisfying duality. On the other hand it was shown in [3] that, in the presence of a field algebra and gauge group, duality is characteristic of sectors corresponding to one-dimensional representations of the gauge group. This suggests a connexion between the statistics of a sector and the dimension of the corresponding representation of the gauge group.

4.1. Theorem. The sector corresponding to $\sigma \in \hat{\mathscr{G}}$ obeys parastatistics of order $\operatorname{dim} \sigma$.

Proof. Let $\varrho$ be an irreducible morphism from $\Delta^{\prime}$. Our aim will be to compute the statistics parameter [2; Theorem 3.7] of $\varrho$ in terms of $d=\operatorname{dim} D(\varrho)$. This computation involves the conjugate sector so let $\bar{\varrho} \in \Delta^{\prime}$ be such that $D(\varrho)$ is a representation conjugate to $D(\varrho)$. Then we have an antiunitary map $\psi \in H(\varrho) \rightarrow \bar{\psi} \in H(\varrho)$, say, intertwining $D(\varrho)$ and $D(\varrho)$. Take $\psi_{i}, i=1,2, \ldots d$ to be an orthonormal basis in $H(\varrho)$ and define

$$
R=\sum_{i=1}^{d} \bar{\psi}_{i} \psi_{i}
$$

so that by the orthonormality of the $\psi_{i}$ we have

$$
R^{*} R=d I .
$$

Using the orthogonality relations for the coefficients of irreducible representations of $\mathscr{G}$, it is easily checked that

$$
m\left(\bar{\psi} \psi^{\prime}\right)=\frac{1}{d}\left(\psi, \psi^{\prime}\right) R, \quad \psi, \psi^{\prime} \in H(\varrho) .
$$

Now $R$ clearly intertwines $\imath$ and $\varrho \varrho$ and since it is also gauge invariant it is an element of $\mathscr{T}(\bar{\varrho} \varrho, l)$. In fact because the identity representation of $\mathscr{G}$ appears with multiplicity one in $D(\varrho) \otimes D(\varrho)$, Theorem 3.6 implies that the vacuum sector appears in the decomposition of $\varrho \varrho$ precisely 
once. Hence every element of $\mathscr{T}(\bar{\varrho} \varrho, l)$ is a multiple of $R$. Similarly

$$
\begin{gathered}
\sum_{i=1}^{d} \psi_{i} \bar{\psi}_{i}=\bar{R} \in \mathscr{T}(\varrho \bar{\varrho}, l), \\
\bar{R}^{*} \bar{R}=d I, \\
m\left(\psi^{\prime} \bar{\psi}\right)=\frac{1}{d}\left(\psi, \psi^{\prime}\right) \bar{R}, \quad \psi, \psi^{\prime} \in H(\varrho) .
\end{gathered}
$$

Since $\psi \in H(\varrho)$ we have

$$
\bar{R}^{*} \varrho(R)=\sum_{i=1}^{d} \bar{\psi}_{i}^{*} R \psi_{i}=\sum_{i, j=1}^{d}\left(\bar{\psi}_{i}, \bar{\psi}_{j}\right) \psi_{j} \psi_{i}^{*}=\sum_{i=1}^{d} \psi_{i} \psi_{i}^{*} .
$$

Now $\sum_{i=1}^{d} \psi_{i} \psi_{i}^{*}=I$, the support of $H(\varrho)$, hence

$$
\bar{R}^{*} \varrho(R)=I
$$

and similarly

$$
R^{*} \varrho(\bar{R})=I
$$

Rewriting (4.4) using the intertwiners $\varepsilon$ introduced in [2; Theorem 4.2] will give us the information we need. In fact since $\mathscr{T}(\varrho \bar{Q}, l)$ is one dimensional $\bar{R}=\mu \varepsilon(\bar{\varrho}, \varrho) R$ where the complex number $\mu$ has modulus one by $(4.2)$ and $\left(4.2^{\prime}\right)$. Also by $[2$; Theorem 4.3$] \varrho(R)=\varepsilon(\varrho \varrho, \varrho) R$ so in place of (4.4) we may write

$$
R^{*} \varepsilon(\varrho, \bar{\varrho}) \varepsilon(\bar{\varrho} \varrho, \varrho) R=\mu I .
$$

However $\varepsilon(\varrho, \varrho) \varepsilon(\varrho \varrho \varrho, \varrho)=\bar{\varrho}\left(\varepsilon_{\varrho}\right)$; this is a consequence of [2; Theorem 4.2] but can also be seen by specializing to the case that $\varrho$ and $\varrho$ are spacelike. Consequently

$$
R^{*} \varrho\left(\varepsilon_{\varrho}\right) R=\mu I .
$$

Comparing with [2; Eqs. (3.13) and (3.16)] we see that the statistics parameter of our sector is given by

$$
\lambda(\varrho)=\mu d^{-1} .
$$

In other words our sector obeys parastatistics of order $d=\operatorname{dim} D(\varrho)$ completing the proof of the theorem.

In the course of the proof we have shown that $\mu=+1$ if $\varrho$ is a para-Bose sector and $\mu=-1$ if $\hat{\varrho}$ is a para-Fermi sector. Consequently

$$
\bar{R}= \pm \varepsilon(\varrho, \varrho) R
$$

where the sign is just the sign of the statistics parameter. 
We remark that the statistical dimension $d(\varrho)$ of a localized morphism $\varrho \in \Delta^{\prime}$ defined in $[2 ; \S \mathrm{VI}]$ is just the dimension of $D(\varrho)$ because $d(\varrho)$ and $\operatorname{dim} D(\varrho)$ agree on irreducibles by Theorem 4.1 and both are additive on direct sums.

We now examine the commutation properties at spacelike separations of fields of irreducible tensor character under the gauge group to try and uncover any intrinsic features determined by the statistics of the sectors involved. It is known for example that if $F_{1}, F_{2}^{*} \in \mathfrak{F}$ are spacelike separated and lead to a simple sector, then

$$
F_{1} F_{2} \mp F_{2} F_{1}=0
$$

according to whether the sector obeys Bose or Fermi statistics. This intrinsic commutation relation has the special feature that the left hand side of (4.6) is anyway gauge invariant and hence in $\mathfrak{A}$. In this sense (4.6) may be generalized to arbitrary sectors:

4.2. Theorem. Let $F_{1} \in \mathfrak{F}\left(\mathcal{O}_{1}\right)$ and $F_{2} \in \mathfrak{F}\left(\mathcal{O}_{2}\right)$ be irreducible tensors of type $\sigma$ and $\bar{\sigma}$ respectively, $\sigma \in \hat{\mathscr{G}}$. If $\mathcal{O}_{1}$ and $\mathcal{O}_{2}$ are spacelike separated double cones we have

$$
\int_{\mathscr{G}} \alpha_{g}\left(F_{1} F_{2} \mp F_{2} F_{1}\right) d \mu(g)=0
$$

where the - or + sign is to be taken according to whether the sector corresponding to $\sigma$ obeys para-Bose or para-Fermi statistics.

Proof. Choose morphisms $\varrho$ and $\varrho$ localized in $\mathcal{O}_{1}$ and $\mathcal{O}_{2}$ respectively and such that $D(\varrho)$ and $D(\bar{\varrho})$ are of class $\sigma$ and $\bar{\sigma}$ respectively. Let $\psi \in H(\varrho) \rightarrow \bar{\psi} \in H(\varrho)$ be an antiunitary map interwining $D(\varrho)$ and $D(\varrho)$. By Proposition 2.2 we can choose $B_{i} \in \mathfrak{A}\left(\mathcal{O}_{i}\right), i=1,2$ and $\psi, \psi^{\prime} \in H(\varrho)$ such that

$$
F_{1}=B_{1} \psi^{\prime}, \quad F_{2}=B_{2} \bar{\psi} .
$$

By our choice of $\varrho$ and $\varrho$ and by locality we have $\varrho\left(B_{2}\right)=B_{2}, \bar{\varrho}\left(B_{1}\right)=B_{1}$ and $B_{1} B_{2}=B_{2} B_{1}$. Hence using (4.3) and (4.3') as well, we get

$m\left(F_{1} F_{2} \mp F_{2} F_{1}\right)=m\left(B_{1} \varrho\left(B_{2}\right) \psi^{\prime} \bar{\psi} \mp B_{2} \varrho\left(B_{1}\right) \bar{\psi} \psi^{\prime}\right)=\frac{1}{d}\left(\psi, \psi^{\prime}\right) B_{1} B_{2}(\bar{R} \mp R)$.

Applying (4.5) and noting that by [2; Theorem 4.2], $\varepsilon(\bar{\varrho}, \varrho)=1$ if $\varrho$ and $\varrho$ are spacelike separated the theorem follows.

Note that if $F_{i} \in \mathfrak{F}$ is an irreducible tensor of type $\sigma_{i}, i=1,2$ the expression (4.7) vanishes identically unless $\sigma_{1} \otimes \sigma_{2}$ contains the identity representation, i.e. unless $\sigma_{2}=\bar{\sigma}_{1}$. 


\section{Wave Function Permutations}

Let $g \rightarrow u_{\sigma}(g)$ be a unitary representation of $\mathscr{G}$ in the class $\sigma \in \hat{\mathscr{G}}$ acting on the $d$-dimensional Hilbert space $\mathscr{H}_{\sigma}$. Given $\varrho \in \Delta^{\prime}$ with $D(\varrho)$ of class $\sigma$ we can define a unitary map $\psi$ of $\mathscr{H}_{\sigma}$ onto $H(\varrho) \subset \mathfrak{F}$ which interwines $u_{\sigma}$ and $D(\varrho)$ :

$$
\boldsymbol{\psi}: v \in \mathscr{H}_{\sigma} \rightarrow \psi(v) \in H(\varrho), \quad \alpha_{g}(\psi(v))=\psi\left(u_{\sigma}(g) v\right)
$$

We can regard $\varrho \in \Delta^{\prime}$ and $v \in \mathscr{H}_{\sigma}$ as the generalized wave function and "isospin" data of the state vector $\psi(v) \Omega$.

Now let $\varrho_{1}, \ldots, \varrho_{n}$ be morphisms in class $\varrho$ localized in mutually spacelike double cones, and $U_{j} \in \mathfrak{A}$ a unitary intertwining operator between $\varrho$ and $\varrho_{j}$

$$
U_{j} \varrho(A)=\varrho_{j}(A) U_{j}, \quad A \in \mathfrak{A} .
$$

We define the unitary mapping $\psi^{j}$ of $\mathscr{H}_{\sigma}$ onto $H\left(\varrho_{j}\right)$ intertwining $u_{\sigma}$ and $D\left(\varrho_{j}\right)$ by

$$
\psi^{j}: v \in \mathscr{H}_{\sigma} \rightarrow \psi^{j}(v)=U_{j} \psi(v) \in H\left(\varrho_{j}\right) .
$$

By Proposition 3.3 the space $\mathscr{H}_{\sigma} \otimes \cdots \otimes \mathscr{H}_{\sigma}(n$ factors) is mapped unitarily onto $H\left(\varrho_{1} \ldots \varrho_{n}\right)$ by the tensor product map

$$
\boldsymbol{\psi}_{p}: v_{1} \otimes \cdots \otimes v_{n} \rightarrow \psi^{p(1)}\left(v_{1}\right) \ldots \psi^{p(n)}\left(v_{n}\right)
$$

where $p \in P^{(n)}$, the permutation group of $n$ objects with identity $e$.

We say that $\boldsymbol{\psi}_{p}$ is obtained from $\boldsymbol{\psi}_{e}$ by a wave function permutation. The full permutation involving the gauge group indices as well is given by

$$
\psi^{p(1)}\left(v_{p(1)}\right) \ldots \psi^{p(n)}\left(v_{p(n)}\right)=\boldsymbol{\psi}_{p} \circ u(p)\left(v_{1} \otimes \cdots \otimes v_{n}\right)
$$

where $p \rightarrow u(p)$ denotes the unitary representation of $P^{(n)}$ on $\mathscr{H}_{\sigma} \otimes \cdots \otimes \mathscr{H}_{\sigma}$ such that $u(p) v_{1} \otimes \cdots \otimes v_{n}=v_{p^{-1}(1)} \otimes \cdots \otimes v_{p^{-1}(n)}$. If $\mathfrak{F}$ were made up of Bose and Fermi fields with normal commutation relations we would have

$$
\psi^{p(1)}\left(v_{p(1)}\right) \ldots \psi^{p(n)}\left(v_{p(n)}\right)= \pm \psi^{1}\left(v_{1}\right) \ldots \psi^{n}\left(v_{n}\right)
$$

where we have a positive sign for a para-Bose sector and sign $(\varrho)$ for a para-Fermi sector. Equivalently

$$
\boldsymbol{\psi}_{p}=\boldsymbol{\psi}_{e} \circ u_{\lambda}(p) \quad \text { (normal case) }
$$

where $u_{\lambda}(p)=u(p)$ if $\lambda=\lambda(\varrho)>0$ and $u_{\lambda}(p)=\operatorname{sign}(p) u(p)$ if $\lambda=\lambda(\varrho)<0$.

5.1. Theorem. The wave function permutations are induced by a unitary representation $V$ of $P^{(n)}$ on $\mathscr{H}_{\sigma} \otimes \cdots \otimes \mathscr{H}_{\sigma}$ :

$$
\boldsymbol{\psi}_{p}=\boldsymbol{\psi}_{e} \circ V(p) .
$$


The representation $V$ depends only on the sector $\hat{\varrho}$ and is unitarily equivalent to $u_{\lambda}$. Further $V$ commutes with the representation of $\mathscr{G}$ on $\mathscr{H}_{\sigma} \otimes \cdots \otimes \mathscr{H}_{\sigma}$.

Proof. One sees directly that $\boldsymbol{\psi}_{p}\left(v_{1} \otimes \cdots \otimes v_{n}\right)=U\left(p^{-1}\right) \psi\left(v_{1}\right) \ldots \psi\left(v_{n}\right)$ where $U\left(p^{-1}\right)=U_{p(1)} \varrho\left(U_{p(2)}\right) \ldots \varrho^{n-1}\left(U_{p(n)}\right)$. Therefore setting $\eta(p)$ $=U\left(p^{-1}\right) U(e)^{*}$ we have

$$
\psi_{p}=\eta(p) \psi_{e}
$$

Comparing with the representation $\varepsilon_{o}^{(n)}$ of $P^{(n)}$ in the $n$-fold product of the sector $\hat{\varrho}, \varepsilon_{\varrho}^{(n)}(p)=U(p)^{*} U(e)[2 ;(4.11)$ and (4.17)], we see that

$$
\eta(p)=U(e) \varepsilon_{\varrho}^{(n)}(p) U(e)^{*}
$$

so that $\eta$ is a unitary representation of $P^{(n)}$. Clearly

$$
\eta(p) \in \mathscr{T}\left(\varrho_{1} \ldots \varrho_{n}, \varrho_{1} \ldots \varrho_{n}\right) .
$$

To prove the Theorem we need

5.2. Lemma. The relation

$$
B \boldsymbol{\psi}_{e}=\boldsymbol{\psi}_{e} \circ \tilde{B}, \quad B \in \mathscr{T}\left(\varrho_{1} \ldots \varrho_{n}, \varrho_{1} \ldots \varrho_{n}\right)
$$

defines $a^{*}$-isomorphism of $\mathscr{T}\left(\varrho_{1} \ldots \varrho_{n}, \varrho_{1} \ldots \varrho_{n}\right)$ onto $\left(u_{\sigma} \otimes \cdots \otimes u_{\sigma}\right)(\mathscr{G})^{\prime}$. Further

$$
\phi(B)=\frac{1}{d^{n}} \operatorname{Tr} \tilde{B} \cdot I
$$

where $\phi=\phi_{n} \ldots \phi_{1}$ and $\phi_{j}$ is the left inverse of $\varrho_{j}, j=1,2 \ldots n$.

Proof. The first assertion follows immediately noting that $\psi_{e}$ is a unitary map of $\mathscr{H}_{\sigma} \otimes \cdots \otimes \mathscr{H}_{\sigma}$ onto $H\left(\varrho_{1} \ldots \varrho_{n}\right)$ and comparing with Proposition 3.5. If $\psi_{1}, \ldots, \psi_{d}$ is an orthonormal basis in $H(\varrho)$, the map

$$
A \in \mathfrak{A} \rightarrow \phi(A)=\frac{1}{d} \sum_{i=1}^{d} \psi_{i}^{*} A \psi_{i}
$$

is a linear positive identity preserving map of $\mathfrak{A}$ into $\mathfrak{A}$ such that $\phi(A \varrho(B))=\phi(A) B, A, B \in \mathfrak{A}$. By [2; Theorem 3.9] if $\varrho$ is irreducible, $\phi$ is the unique left inverse of $\varrho$. We may take $\psi_{j}=\psi\left(e_{j}\right)$ where $e_{1}, \ldots, e_{d}$ is an orthonormal basis in $\mathscr{H}_{\sigma}$. We then have by (5.8)

$$
\begin{aligned}
\operatorname{Tr} \tilde{B} & =\sum_{i_{1}, \ldots i_{n}}\left(e_{i_{1}} \otimes \cdots \otimes e_{i_{n}}, \tilde{B} e_{i_{1}} \otimes \cdots \otimes e_{i_{n}}\right) \\
& =\sum_{i_{1}, \ldots i_{n}} \psi^{n}\left(e_{i_{n}}\right)^{*} \ldots \psi^{1}\left(e_{i_{1}}\right)^{*} B \psi^{1}\left(e_{i_{1}}\right) \ldots \psi^{n}\left(e_{i_{n}}\right)
\end{aligned}
$$

and comparing with (5.10) we get (5.9). 
Remark. We note in passing the relations

$$
\begin{aligned}
m\left(\psi^{*} A \psi^{\prime}\right)= & \left(\psi, \psi^{\prime}\right) \phi(A) \\
m\left(\psi^{\prime} A \psi^{*}\right)= & \frac{1}{d}\left(\psi, \psi^{\prime}\right) \varrho(A) \\
& A \in \mathfrak{U}, \psi, \psi^{\prime} \in H(\varrho) .
\end{aligned}
$$

The first of these generalizes (5.10).

To complete the proof of Theorem 5.1 define $V(p)=\tilde{\eta}(p)$ and note that (5.5) follows trivially from (5.6) and (5.8). If $\phi_{0}$ is the left inverse of $\varrho$ then from (5.7) and [2; Eq. (5.1) and Lemma 5.4]

$$
\phi(\eta(p))=\phi_{0}^{n}\left(\varepsilon_{\varrho}^{(n)}(p)\right)=\omega_{\lambda}^{n}(p)=\frac{1}{d^{n}} \operatorname{Tr} u_{\lambda}(p) .
$$

Hence $V$ and $u_{\lambda}$ have the same trace by (5.9) and so must be equivalent. The matrix elements of $V(p)$ in the basis $e_{i_{1}} \otimes \cdots \otimes e_{i_{n}}$ are given by

$$
\left(e_{i_{1}} \otimes \cdots \otimes e_{i_{n}}, V(p) e_{j_{1}} \otimes \cdots \otimes e_{j_{n}}\right)=\psi_{i_{n}}^{*} \ldots \psi_{i_{1}}^{*} \varepsilon_{\varrho}^{(n)}(p) \psi_{j_{1}} \cdots \psi_{j_{n}}
$$

where we have used (5.1') and (5.7). If $\boldsymbol{W}=\left(\varrho^{\prime}|W| \varrho\right)$ is a unitary intertwiner, then replacing $\varrho$ by $\varrho^{\prime}$ and $\psi_{j}$ by $W \psi_{j}$ leaves (5.13) unchanged by [2; Eq. (4.18)]. Hence $V$ depends only on the sector $\hat{\varrho}$.

Remark. Let $K$ be a unitary on $\mathscr{H}_{\sigma} \otimes \cdots \otimes \mathscr{H}_{\sigma}$ such that

$$
K u_{\lambda}(p)=V(p) K, \quad p \in P^{(n)}
$$

so that by (5.5)

$$
\boldsymbol{\psi}_{p} \circ K=\boldsymbol{\psi}_{e} \circ K u_{\lambda}(p)
$$

If $K$ were induced by a Klein transformation $\psi \in \mathfrak{F} \rightarrow \tilde{\psi} \in \tilde{F}$, i.e. if

$$
\tilde{\psi}^{p(1)}\left(v_{1}\right) \ldots \tilde{\psi}^{p(n)}\left(v_{n}\right)=\boldsymbol{\psi}_{p}{ }^{\circ} K\left(v_{1} \otimes \cdots \otimes v_{n}\right), \quad v_{1}, \ldots v_{n} \in \mathscr{H}_{\sigma}
$$

then by (5.14) fields of irreducible tensor character $\sigma$ in $\tilde{\mathscr{F}}$ would have normal commutation relations.

Theorem 4.2 described an intrinsic feature of the commutation relations between spacelike separated fields transforming according to conjugate representations of $\mathscr{G}$. Theorem 5.1 has given us an intrinsic feature of the commutation relations between spacelike fields transforming according to the same representation of $\mathscr{G}$. The wave function permutations are described by the unitary representation $V$ of $P^{(n)}$ whose equivalence class is determined by the statistics parameter $\lambda$.

We conclude with some remarks on the universality of the concept of statistics for which Theorem 5.1 provides further evidence. There are two ingredients involved in statistics; the first is the more primitive 
concept of a product of states. Here we have two choices, we may use the product of localized states as is done in [2] or we may use the asymptotic product of scattering states and so arrive at particle statistics. Which product we take will have no effect on the statistics assigned to a sector because scattering theory relates the asymptotic product to the product of localized states whose localization centres are far apart. The second ingredient is to define a product of state vectors. In [2] as in elementary wave mechanics the product of state vectors is chosen to be compatible with the product of states. Permuting the order of the product of state vectors provides a representation $\varepsilon_{\varrho}^{(n)}$ of the permutation group commuting with the observables.

However in the presence of a field algebra it is customary to define a product of state vectors in terms of a product of field operators.

$$
\psi_{1} \Omega \times \psi_{2} \Omega=\psi_{1} \psi_{2} \Omega .
$$

In this way the statistics is analyzed in terms of the commutation relations of fields. Setting

we have

$$
|p\rangle=\psi^{p(1)}\left(v_{1}\right) \ldots \psi^{p(n)}\left(v_{n}\right) \Omega
$$

$$
\eta(p)|q\rangle=|p q\rangle, \quad p, q \in P^{(n)}
$$

so that the wave function permutations are unitarily implemented. However since $\eta(p) \in \mathfrak{U}$ we see that unless we have ordinary Bose or Fermi statistics the state vectors $|q\rangle$ do not represent the same state over $\mathfrak{A}$. In other words the product of these field operators does not induce the commutative product of the corresponding localized states. On the other hand by Theorem 5.1, the wave function permutations yield the correct statistics. The consistency of the second-quantized version of parastatistics involving wave function permutations and the first quantized version involving permutations commuting with the observables was first shown by Stolt and Taylor [5]. They remarked that these two kinds of permutations were implemented in different Hilbert space representations where the same states were represented by spaces of state vectors of different multiplicity. The same feature can be seen here: $\varepsilon_{e}^{(n)}(p)$ operates in the representation $\pi_{0}{ }^{\circ} \varrho^{n}$ and $\eta(p)$ in the defining representation on $\mathscr{H}$ where the multiplicities are in general quite different. The wave function permutations $|q\rangle \rightarrow|p q\rangle$ are precisely the permutations which arise in [6] in comparing theories described in terms of para-Fermi fields with theories described as here in terms of a field algebra and a gauge group. These wave function permutations arise too in the description of parastatistics in [7] in terms of "distinct but dynamically indistinguishable particles". This discussion indicates the basic compatibility of the various approaches to parastatistics. 
Acknowledgements. We wish to thank Rudolf Haag for numerous fruitful discussions. We would like to acknowledge the hospitality of the Centre Universitaire de MarseilleLuminy in one stage of our collaboration; J. E. Roberts wishes to thank the Istituto di Fisica dell'Università di Roma for the hospitality in one stage of this work.

\section{References}

1. Doplicher, S., Haag, R., Roberts, J. E.: Fields, observables and gauge transformations II. Commun. math. Phys. 15, 173-200 (1969).

2. Doplicher, S., Haag, R., Roberts, J.E.: Local observables and particle statistics I. Commun. math. Phys. 23, 199-230 (1971).

3. Doplicher, S., Haag, R., Roberts, J. E.: Fields, observables and gauge transformations I. Commun. math. Phys. 13, 1-23 (1969).

4. Mac Lane, S.: Categories for the working mathematician. Berlin-Heidelberg-New York: Springer 1971.

5. Stolt, R.A., Taylor, J. R.: Parastatistics. Nucl. Phys. B 19, 1-19 (1970).

6. Drühl,K., Haag, R., Roberts,J.E.: On parastatistics. Commun. math. Phys. 18, 204-226 (1970).

7. Landshoff, P.V., Stapp, H.P.: Parastatistics and a unified theory of identical particles. Ann. Phys. 45, 72-92 (1967).

S. Doplicher

Istituto di Fisica

"Guglielmo Marconi"

Piazzale delle Scienze 5

Roma

Italy

\author{
J. E. Roberts \\ II. Institut für Theoretische Physik \\ der Universität \\ D-2000 Hamburg 50 \\ Luruper Chaussee 149 \\ Federal Republic of Germany
}

